\title{
Squared Weyl and Dirac fields with the Sommers-Sen connection associated with $V_{4}^{3}$ distributions
}

\author{
V.Pelykh \\ Pidstryhach Institute for Applied Problems in Mechanics \& Mathematics \\ the National Academy of Sciences of Ukraine \\ $3^{\sigma}$ Naukova St., UA-290601 Lviv, Ukraine
}

Received February 10, 1998

Generalization of the Sommers-Sen spinor connection for spinor fields, associated with the distribution $V_{4}^{3}$ is made and on its basis the equations for Weyl and Dirac null vector fields on complexificated $V_{4}^{3}$ are obtained. We interpret the obtained results by examining the interaction of spinor fields with inertial forces.

Key words: spinor, manifold, distribution, null field

PACS: 04.20.- $q, 02.40 . K y$

\section{Introduction}

As it is known, the four-dimensional description of relativistic fields for a number of problems, especially for the comparison of theoretical provisions and experimental results, must be substituted by a $3+1$ description. For the spinor fields the original method of their description in the $3+1$ form was proposed in [1] and developed in [2]. The obtained in [2] Sen-Witten equation is widely used in the gravitational field energy problem investigations [3-5]. But this does not allow us to study all the variety of physical effects in the interactions of spinor fields with inertial forces, because this method is based on the foliation of curved space-time by space-like hypersurfaces and thus on nonrotatory frames of reference. In our work [6] we introduced a covariant derivative of spinor fields associated with the space-like distribution $V_{4}^{3}$, which generalizes the Sommers-Sen covariant derivative and obtained on this basis a $3+1$ equation for Weyl and Rarita-Schwinger fields in arbitrary frames of reference, not only in nonrotatory ones. In this work we obtain for an arbitrary frame of reference the equations for a complex 3-vector, which are correspondent to Weyl spinors and bispinors. These squared equations of Weyl and Dirac fields are, in fact, a 3+1 splitting of the Penrose-Rindler [7] tensor form of spinor differential equations. 
In section 2 we briefly review the technique for obtaining $3+1$ spinor equations with a spinor connection on nonintegrable manifolds. In section 3 we give a spinor representation of tensor fields on the $V_{4}^{3}$ distribution.

\section{Generalization of the Sommers-Sen derivative on distribu- tions}

Let us consider the oriented manifold $V_{4}$ of class $C^{\infty}$ which is noncompact or of a zero Euler characteristic; then it accepts the Lorentz $\mathbf{g}$ metric. Let us denote, as usual, a tangent bundle over $V_{4}$ by $T V_{4}$.

Definition 1. A vector subbundle $V_{4}^{m}, \quad 1 \leqslant m \leqslant 3$, of $T V_{4}$ is called a distribution over $V_{4}$. Let us denote by $C^{\infty}\left(V_{4}\right)$ a ring of functions of a $C^{\infty}$ class and by $\Gamma\left(V_{4}^{m}\right)$ - a $C^{\infty}$ module of distribution $V_{4}^{m}$ over $V_{4}$.

Definition 2. The mapping

$$
\begin{gathered}
\Gamma\left(V_{4}^{m}\right) \times \Gamma\left(V_{4}^{m}\right) \longrightarrow \Gamma\left(V_{4}^{m}\right), \\
\left(\mathbf{X}, \mathbf{X}_{1}\right) \longrightarrow \mathcal{D}_{\mathbf{X}} \mathbf{X}_{1}, \quad \mathbf{X}, \mathbf{X}_{\mathbf{1}} \in \Gamma(S),
\end{gathered}
$$

which for arbitrary vectors $\mathbf{X}, \mathbf{X}_{1}$ and functions $g, f \in C^{\infty}\left(V_{4}\right)$ satisfies the conditions

$$
\begin{gathered}
\mathcal{D}_{\mathbf{X}}\left(\mathbf{X}_{\mathbf{1}}+\mathbf{X}_{2}\right)=\mathcal{D}_{\mathbf{X}} \mathbf{X}_{1}+\mathcal{D}_{\mathbf{X}} \mathbf{X}_{2} \\
\mathcal{D}_{\mathbf{X}}\left(f \mathbf{X}_{\mathbf{1}}\right)=\mathbf{X}(f) \mathbf{X}_{1}+f \mathcal{D}_{\mathbf{X}} \mathbf{X}_{1} \\
\mathcal{D}_{f \mathbf{X}+g \mathbf{X}_{1}} \mathbf{X}_{2}=f \mathcal{D}_{\mathbf{X}} \mathbf{X}_{2}+g \mathcal{D}_{\mathbf{X}_{1}} \mathbf{X}_{2}
\end{gathered}
$$

is called a covariant derivative or anholonomic connection on the distribution $V_{4}^{m}$.

The homomorphism

$$
\Gamma\left(B_{0}\right): \quad \Gamma\left(T V_{3}\right) \longrightarrow \Gamma\left(V_{4}^{m}\right)
$$

of cross-sections is correspondent to projections $B_{0}$ of bundle $T V_{4}$ on subbundle $V_{4}^{m}$.

Definition 3. $i m C_{0}$, where $C_{0}=1_{T V_{4}}-B_{0}$, is called rigging $V_{4}^{4-m}$ of the distribution $V_{4}^{m} \subset T V_{4}$.

Let $V_{4}^{1}$ be a one-dimensional time-like distribution over $V_{4}$; its unitary crosssection $\mathbf{u}$ is identified with the field of 4 -velocity of some frame of reference, and the integral curve of cross-section $\mathbf{u}$ - with its time lines. The normal rigging $V_{4}^{3}$ of the distribution $V_{4}^{1}$ is a geometrical image of the physical space for the appropriate frame of reference and is nonintegrable in general. The vectors, which belong to $V_{4}^{1}$, are called time vectors, and those belonging to $V_{4}^{3}$ - spatial vectors.

Further we require that the second Stiefel-Whitney class $w_{2}$ of manifold $V_{4}$ equal zero. Then $V_{4}$ permits the $S L(2, C)$ spinor structure. Let us denote by $\mathcal{S}_{r, s}\left(V_{4}\right)$ the $C^{\infty}$ module of spinor fields of $(r, s)$ valence on $V_{4}$. 
Let us introduce the $C^{\infty}$ module of $\mathcal{S}_{r, s}\left(V_{4}^{3}\right)$ spinor fields of $(r, s)$ valence on $V_{4}$ associated with the distribution $V_{4}^{3}$ in the following way: its elements are spinor fields of the form $\varphi T^{A \ldots L}{ }_{M \ldots Q}$ and

$$
\begin{aligned}
\psi T^{A \ldots K C \ldots L}{ }_{M \ldots R P \ldots Q} \ldots & \left(\sqrt{2} u_{\dot{A}}^{A}\right) \ldots\left(\sqrt{2} u_{\dot{K}}^{K}\right)\left(\sqrt{2} u_{M}^{\dot{M}}\right) \ldots\left(\sqrt{2} u_{R}^{\dot{R}}\right) \times \\
& T^{\dot{A} \ldots \dot{K} C \ldots L}{ }_{\dot{M} \ldots \dot{R} P \ldots Q} .
\end{aligned}
$$

The defined in such a way $C^{\infty}$ module of $\mathcal{S}_{r, s}\left(V_{4}^{3}\right)$ is a module of $S U(2)$ spinor fields. Let us call the module $\mathcal{S}_{r, s}\left(V_{4}^{3}\right)$ with the basis limited to the hypersurface $\Sigma$ a module of $S U(2)$ spinor fields on the anholonomic hypersurface $\Sigma$. In a particular case, when this hypersurface is ordinary and space-like, the module of $S U(2)$ spinor fields on it coincides with the module of Sommers-Sen spinor fields.

We introduce the antisymmetric tensor $\mathbf{A}$ of anholonomicity of $V_{4}^{3}, \mathbf{A} \in V_{4}^{3}$. Let

$$
\begin{gathered}
\mathbf{T}: \Gamma\left(V_{4}^{3}\right) \times \Gamma\left(V_{4}^{3}\right) \longrightarrow \Gamma(P), \\
\mathbf{T}=\Gamma\left(C_{0}\right)\left[\mathbf{X}_{1}, \mathbf{X}_{2}\right] ; \quad \mathbf{X}_{\mathbf{1}}, \mathbf{X}_{\mathbf{2}} \in \Gamma(P) .
\end{gathered}
$$

Then $\mathbf{T}=4 \mathbf{A} \otimes \mathbf{u}$. In the coordinate basis on some open domain in $V_{4}$ tensor $\mathbf{A}$ has the components [8]

$$
A_{\mu \lambda}=\frac{1}{2} h_{\mu}^{\nu} h_{\lambda}^{\delta} u_{[\nu} u_{\delta]} .
$$

Let us introduce a spatial covariant derivative for spinor fields associated with the distribution $V_{4}^{3}$ as the mapping

$$
\begin{gathered}
\Gamma\left(V_{4}^{3}\right) \times \mathcal{S}_{1,0}\left(V_{4}^{3}\right) \longrightarrow \mathcal{S}_{1,0}\left(V_{4}^{3}\right), \\
\left(X_{A B}, \lambda_{C}\right) \longrightarrow \mathcal{D}_{A B} \lambda_{C}, \quad X_{A B} \in \mathcal{S}_{1,0}\left(V_{4}^{3}\right) \times \mathcal{S}_{1,0}\left(V_{4}^{3}\right),
\end{gathered}
$$

determined by the condition

$$
\mathcal{D}_{A B} \lambda_{C}=\sqrt{2} u_{(A}{ }^{\dot{A}} \nabla_{B) \dot{A}} \lambda_{C}-\frac{1}{\sqrt{2}}\left(\pi_{A B C}{ }^{D}+A_{A B C}{ }^{D}\right) \lambda_{D}
$$

where $\nabla_{B \dot{A}}$ is a spinor representation of an operator of the covariant derivative on $V_{4}$, in agreement with the metrical connection. The action $\mathcal{D}_{A B}$ on spinors of a higher valence extends in accordance with the Leibnitz rule and the action on vector fields satisfies the condition (1)-(3).

Reducing the $S L(2, C)$ operator of the covariant derivative to the $S U(2)$ operator, we obtain:

$$
\nabla_{A B}=\sqrt{2}\left(u_{[B}^{\dot{A}} \nabla_{A] \dot{A}}+u_{(B}^{\dot{A}} \nabla_{A) \dot{A}}\right)=\frac{\sqrt{2}}{2} \varepsilon_{A B} u^{A \dot{A}} \nabla_{A \dot{A}}+\sqrt{2} n_{(B}^{\dot{A}} \nabla_{A) \dot{A}}
$$


The first term denoted by $\frac{\sqrt{2}}{2} \varepsilon_{A B}(\mathbf{u} \cdot \nabla)$ is a time derivative, the second one is represented in terms of space derivative $\mathcal{D}_{A B}$ in the rigging. Finally, we obtain the action of $\nabla_{A B}$ on spinor $\lambda_{C}$ in the form:

$$
\nabla_{A B} \lambda_{C}=\frac{\sqrt{2}}{2} \varepsilon_{A B} u^{A \dot{A}} \nabla_{A \dot{A}} \lambda_{C}+\mathcal{D}_{A B} \lambda_{C}-\frac{\sqrt{2}}{2}\left(\pi_{A B}{ }^{C}{ }_{D}+A_{A B}{ }^{C}{ }_{D}\right) \lambda^{D} .
$$

We obtain the generalized $3+1$ form of the Weyl equation

$$
\nabla_{A \dot{A}} \lambda^{A}=0
$$

carrying out the $S L(2, C) \rightarrow S U(2)$ reduction and using (6). Then we have

$$
(\mathbf{u} \cdot \nabla) \lambda_{A}+\sqrt{2} \mathcal{D}_{A B} \lambda^{B}+\frac{1}{2} \pi \lambda_{A}-A_{B A}{ }^{B}{ }_{D} \lambda^{D}=0 .
$$

Therefore, the Weyl spinor $\lambda_{A} \in \mathcal{S}_{1,0}\left(V_{4}^{3}\right)$ is determined by the geometric properties of $V_{4}$ and by both the geometric and equally physical properties of $V_{4}^{3}$. These properties are determinated by the acceleration spinor $F_{A B} \in \mathcal{S}_{2,0}\left(V_{4}^{3}\right)$, the angular velocity spinor $A_{A B C D} \in \mathcal{S}_{4,0}\left(V_{4}^{3}\right)$ and the rate-of-strain spinor $\pi_{A B C D} \in \mathcal{S}_{4,0}\left(V_{4}^{3}\right)$. These spinors are uniquely expressed by the Schouten first order curvature tensors of $V_{4}^{3}$ and vector $\mathbf{u} \in V_{4}^{1}$.

\section{Spinor representation of tensor fields on the $V_{4}^{3}$ distribu- tions}

Let spinor field $\mathbf{T} \in S_{2 r, 2 s}\left(V_{4}^{3}\right)$. If $\mathbf{T}$ is symmetric in all pairs of indices, then $\mathbf{T} \in V_{4}^{3}$. The projector from $T V_{4}$ into $V_{4}^{1}$ is $\mathbf{u} \otimes \mathbf{u}$, the projector from $T V_{4}$ into $V_{4}^{3}$ is $\mathbf{h}=\mathbf{g}+\mathbf{u} \otimes \mathbf{u}$.

It is easy to characterise the $S U(2)$ representation of a space projected tensor

$$
\begin{gathered}
\bar{v}_{A B}=\bar{v}_{A \dot{A}} \sqrt{2} u_{B}^{\dot{A}}=v_{\nu}\left(g_{\mu}^{\nu}-u^{\nu} u_{\mu}\right) h_{m}^{\mu} \sigma_{A \dot{A}}^{m}=v_{\nu}\left(g_{\mu}^{\nu}-u^{\nu} u_{\mu}\right) \sigma_{A \dot{A}}^{\mu}= \\
\bar{v}_{\mu} \sigma_{A \dot{A}}^{\mu} \sqrt{2} u_{B}^{\dot{A}}=v_{m} \sigma_{A B}^{m}=v_{\mu} \sigma_{A B}^{\mu} .
\end{gathered}
$$

The overline denotes the components of space projected tensors, the symbol ${ }^{\wedge}$ denotes the components of time projected tensors, $\sigma_{A \dot{A}}^{\mu}$ are the Pauli spin matrices and the unit matrix which are referred to as a space-time tetrad. The $\sigma_{A B}^{\lambda}$ and $\sigma_{A B}^{l}$ matrices are given by the formulas

$$
\sigma_{A B}^{l}=\sigma_{A \dot{A}}^{l} \sqrt{2} u_{B}^{\dot{A}}=\sqrt{2} u^{m} \sigma_{A \dot{A}}^{l} \sigma_{m}^{B \dot{A}}
$$

and, respectively,

$$
\sigma_{A B}^{\lambda}=\sigma_{A \dot{A}}^{\lambda} \sqrt{2} u_{B}^{\dot{A}}=\sqrt{2} u^{\mu} \sigma_{A \dot{A}}^{\lambda} \sigma_{\mu}^{B \dot{A}} .
$$


For the matrices $\sigma_{A B}^{\lambda}$ we obtain the necessary in the following consideration identities which substitute for the normalization and orthogonalization identities of the Pauli matrices:

$$
\begin{aligned}
\sigma_{A B}^{\lambda}{ }_{\lambda}{ }^{D C}=\sigma_{A B}^{l} \sigma_{l}{ }^{D C} & =\sqrt{2} n^{\mu} \sigma^{\mu}{ }_{B}{ }^{\dot{A}} \sigma^{\lambda}{ }_{A \dot{A}} \sqrt{2} n^{\nu} \sigma_{\nu}{ }^{C \dot{C}} \sigma^{\lambda D \dot{C}}=-2 \sqrt{2} n^{\nu} \sigma_{\nu}{ }^{C}{ }_{B} \delta_{A}^{D}= \\
& -2 \sqrt{2} \widehat{\sigma}_{\nu}{ }^{C}{ }_{B}, \quad \sigma^{\lambda}{ }_{A B} \sigma_{\mu}{ }^{A B}=4 \delta_{\mu}^{\lambda} .
\end{aligned}
$$

Let us also obtain the necessary for further investigations tensor representation of the spinor form

$$
\bar{y}_{C(A} \bar{z}_{B)}{ }^{C}=\sum_{m=0}^{3} \bar{y}_{m} \sigma_{C(A}^{m} \bar{z}_{m} \sigma_{B) C}^{m}+\sum_{m \neq l}^{3} \bar{y}_{m} \bar{z}_{l} \sigma_{C\left(A^{m}{ }^{m}{ }_{B} C\right.} \cdot
$$

We obtain the matrices $\sigma_{C A}^{m}$ in the form

$$
\sigma_{C A}^{m}=\sqrt{2}\left(n^{0} \omega^{m}{ }_{C A}+n^{1} \tau^{m}{ }_{C A}+n^{2} \chi_{C A}^{m}+n^{3} \psi_{C A}^{m}\right), \quad \sigma_{C}^{l}{ }^{B}=\sigma_{C A}^{l} \varepsilon^{B C},
$$

where

$$
\begin{gathered}
\omega^{m}{ }_{C A}=\sigma_{C \dot{C}}^{m} \sigma_{0 A} \dot{C}, \tau^{m}{ }_{C A}=\sigma_{C \dot{C}}^{m} \sigma_{1 A}{ }^{\dot{C}}, \chi^{m}{ }_{C A}=\sigma_{C \dot{C}}^{m} \sigma_{2 A} \dot{C} \\
\psi_{C A}^{m}=\sigma_{C \dot{C}}^{m} \sigma_{3 A} \dot{C} .
\end{gathered}
$$

The first sum in (8) equals zero, therefore, further we consider only the second sum in which we distinguish the terms with the products $\bar{y}_{1} \bar{z}_{2}$. In this case the products of the necessary matrices give:

$$
\begin{aligned}
& \omega^{1}{ }_{C A} \omega^{2}{ }_{B}{ }^{C}=-i \omega^{3}{ }_{A B}, \quad \tau^{1}{ }_{C A} \omega^{2}{ }_{B}{ }^{C}=-i \tau^{3}{ }_{A B}, \\
& \omega^{1}{ }_{C A} \tau^{2}{ }_{B}{ }^{C}=\chi_{A B}^{1}, \quad \tau^{1}{ }_{C A} \tau^{2}{ }_{B}{ }^{C}=i \tau^{3}{ }_{A B} \text {, } \\
& \omega^{1}{ }_{C A} \chi^{2}{ }_{B}{ }^{C}=-i \chi_{A B}^{3}, \quad \tau_{C A}^{1} \chi_{B}^{2}{ }_{B}=-\chi_{A B}^{2} \text {, } \\
& \omega^{1}{ }_{C A} \psi^{2}{ }_{B}{ }^{C}=-i \psi^{3}{ }_{A B} ; \quad \tau^{1}{ }_{C A} \psi^{2}{ }_{B}{ }^{C}=-\psi^{2}{ }_{A B} \text {; } \\
& \chi_{C A}^{1} \omega^{2}{ }_{B}^{C}=-i \chi_{A B}^{3}, \quad \psi_{C A}^{1} \omega_{B}^{2}{ }_{B}^{C}=\omega_{A B}^{0} \text {, } \\
& \chi_{C A}^{1} \tau^{2}{ }_{B}{ }^{C}=-\tau_{A B}^{1}, \quad \psi_{C A}^{1} \tau^{2}{ }_{B}{ }^{C}=i \tau_{A B}^{0} \text {, } \\
& \chi_{C A}^{1} \tau_{B}^{2}{ }_{B}^{C}=i \psi_{A B}^{0}, \quad \psi_{C A}^{1}{ }_{C A} \chi_{B}{ }_{B}^{C}=-i \psi_{A B}^{1} \text {, } \\
& \chi^{1}{ }_{C A} \psi^{2}{ }_{B}{ }^{C}=-\psi^{2}{ }_{A B} ; \quad \psi^{1}{ }_{C A} \psi^{2}{ }_{B}{ }^{C}=i \psi^{0}{ }_{A B} \text {. }
\end{aligned}
$$

Then, we obtain the term with $\bar{y}_{1} \bar{z}_{2}$ in the form:

$$
\sqrt{2} \bar{y}_{1} \bar{z}_{2}\left[-i n^{0} \sigma_{(A B)}^{3}+i n^{3} \sigma_{(A B)}^{0}\right] .
$$

By analogy, for terms with $\bar{y}_{2} \bar{z}_{1}$ we have

$$
\sqrt{2} \bar{y}_{2} \bar{z}_{1}\left[i n^{0} \sigma_{(A B)}^{3}-i n^{3} \sigma_{(A B)}^{0}\right] \text {. }
$$


Summing all the terms of the form $\bar{y}_{m} \bar{z}_{l} \quad(m \neq l)$ we find that

$$
\bar{y}_{C(A} \bar{z}_{B)}{ }^{C}=-i \sqrt{2} \varepsilon^{a s n}{ }_{k} n_{a} \bar{y}_{s} \bar{z}_{n} \sigma_{(A B)}^{k} .
$$

Let us consider the evolution of the complex null spatial vector field $\mathbf{L}=-\lambda_{A} \lambda_{B}$ corresponding to the Weyl spinor field. Then, as the first step we obtain

$$
(\mathbf{n} \cdot \nabla) L_{a}=2 \sqrt{2} \lambda_{(B} D_{A) C} \lambda^{C}-\pi L_{a}+2 \lambda_{(B} A_{A) C}{ }^{C}{ }_{D} \lambda^{D} .
$$

Using the Sommers identity

$$
\lambda^{C} D_{A B} \lambda_{C}=\lambda^{C} D_{C(A} \lambda_{B)}-\lambda_{(A} D_{B) C} \lambda^{C}
$$

we have

$$
(\mathbf{n} \cdot \boldsymbol{\nabla}) L_{a}=-\sqrt{2} \lambda^{C} D_{A B} \lambda_{C}-\pi L_{a}+\sqrt{2} D_{C(A} L_{B)}{ }^{C}+2 \lambda_{(B} A_{A) C}{ }^{C}{ }_{D} \lambda^{D} .
$$

For the vector representation of $\sqrt{2} D_{C(A} L_{B)}{ }^{C}$ Sommers [1] applies expression (9) by direct substitution of $\bar{y}_{C A}=D_{C A}$ into it, instead of $\bar{y}_{C A}$. But $D_{l} \sigma_{C A}^{l} \neq 0$, and taking into account this circumstance we obtain:

$$
\begin{gathered}
D_{C(A} L_{B)}^{C}=\sigma_{C(A}^{l} D_{l}\left[\bar{z}_{m} \sigma_{B)}^{m}\right]=D_{l}\left[\sigma_{C(A}^{l} \sigma_{B)}^{m} L_{m}\right]- \\
-L_{m} \sigma^{m}{ }_{(B}^{C} D_{l} \sigma^{l}{ }_{A) C}=-i \sqrt{2} \varepsilon^{a s d}{ }_{k} L_{d} \sigma^{k}{ }_{(A B)} D_{s} n_{a}-i \sqrt{2} \varepsilon^{a s n}{ }_{k} n_{a} \sigma^{k}{ }_{(A B)} D_{s} L_{n}- \\
\sqrt{2} L_{m} F_{r} \sigma_{(B}^{m C} \sigma_{A) C}^{r}-2 \sqrt{2} \varepsilon^{a s d}{ }_{k} n_{a} w_{s} L_{d} \sigma_{(A B)}^{k}-\frac{\sqrt{2}}{2} \pi L_{m} \sigma^{k}{ }_{(A B)}- \\
\sqrt{2} L_{m} \sigma_{(B}^{m C} \sigma_{|C \dot{C}|}^{l} \sigma_{r A)}{ }^{\dot{C}}\left(A^{r}{ }_{l}-D^{r}{ }_{l}\right)+i \sqrt{2} \varepsilon^{a s n}{ }_{k} A_{a s} L_{n} \sigma^{k}{ }_{(A B)}+ \\
2 i \varepsilon^{a s n}{ }_{k} n_{a} F_{s} L_{n} \sigma_{(A B)}^{k} .
\end{gathered}
$$

The first term on the left can be re-expressed [1] as $\widetilde{\mathbf{L}}^{a} D_{a} L_{c}$, where the unit real spatial vector $\widetilde{\mathbf{L}}$ is in the direction of propagation of the neutrino field and is

$$
\widetilde{L}^{c}=-i\left(L_{d} \bar{L}^{d}\right)^{-1} \varepsilon^{a}{ }_{s n k} n_{a} L^{s} L^{n} .
$$

Finally, we find that the squared neutrino equation on $V_{4}^{3}$ distributions, i.e. in an arbitrary frame of reference is

$$
\begin{gathered}
<\mathbf{d} \mathbf{L}, \mathbf{n}>=-\pi \mathbf{L}+2 \cdot 3 ! i *(\mathbf{u} \wedge \mathbf{D} \wedge \mathbf{L})+<\widetilde{\mathbf{L}}, \mathbf{D L}>+ \\
4 \cdot 3 ! *(\mathbf{u} \wedge \mathbf{w} \wedge \mathbf{L})-2 \cdot 3 ! i *(\mathbf{u} \wedge \mathbf{F} \wedge \mathbf{L})-\sqrt{2} \cdot 3 ! i *(\mathbf{A} \wedge \mathbf{L}) .
\end{gathered}
$$

With the help of the squared Weyl equations we can obtain the squared Dirac equation in the $3+1$ form. The Dirac equation is equivalent to the pair of equations

$$
\nabla_{A \dot{A}} \xi^{A}=\frac{m}{\sqrt{2}} \eta_{\dot{A}} \quad \text { and } \quad \nabla^{A \dot{A}} \eta_{\dot{A}}=-m \sqrt{2} \xi^{A}
$$


or in terms of $\xi^{A} \in \mathcal{S}_{0,1}\left(V_{4}^{3}\right), \eta_{B} \in \mathcal{S}_{1,0}\left(V_{4}^{3}\right):$

$$
\nabla_{A B} \xi^{A}=\frac{m}{\sqrt{2}} \eta_{B} \quad \text { and } \quad \nabla^{A B} \eta_{B}=-\frac{m}{\sqrt{2}} \xi^{A}
$$

Let $\mathbf{X}=-\xi_{A} \xi_{B}$ and $\mathbf{Y}=-\eta_{A} \eta_{B}$ be two complex null spatial vector fields. Then, the squared Dirac equation, which describes the evolutionof $\mathbf{X}$ and $\mathbf{Y}$ fields and is written in terms of the tensors determined on the distribution and in the rigging, is the system of two equations:

$$
\begin{gathered}
<\mathbf{d X}, \mathbf{u}>=-\pi \mathbf{X}-2 \cdot 3 ! i *(\mathbf{u} \wedge \mathbf{D} \wedge \mathbf{X})+<\widetilde{\mathbf{X}}, \mathbf{D X}>+ \\
2 *(\mathbf{u} \wedge \mathbf{w} \wedge \mathbf{X})-i *(\mathbf{u} \wedge \mathbf{F} \wedge \mathbf{X})-\sqrt{2} i *(\mathbf{A} \wedge \mathbf{X})+ \\
\sqrt{2} i m<\mathbf{X}, \mathbf{Y}>^{-1 / 2} *(\mathbf{u} \wedge \mathbf{X} \wedge \mathbf{Y})
\end{gathered}
$$

and

$$
\begin{gathered}
<\mathbf{d} \mathbf{Y}, \mathbf{u}>=-i *(\mathbf{u} \wedge \mathbf{D} \wedge \mathbf{Y})-<\tilde{\mathbf{Y}}, \mathbf{D Y}>-6 *(\mathbf{u} \wedge \mathbf{w} \wedge \mathbf{Y})+ \\
i *(\mathbf{u} \wedge \mathbf{F} \wedge \mathbf{Y})-\sqrt{2} i *(\mathbf{A} \wedge \mathbf{X})+\sqrt{2} i m<\mathbf{X}, \mathbf{Y}>^{-1 / 2} *(\mathbf{u} \wedge \mathbf{X} \wedge \mathbf{Y}) .
\end{gathered}
$$

\section{Discussion}

The proposed in [6] and in this paper method for the investigation of an interaction between spinor fields and inertial forces requires the use of a nonintegrable subbundle. Unlike $[1,2]$, the spinor derivatives are determined here by the intrinsic geometry of distribution. This is defined by the physical sense of the problem and not by the application of the tetrad formalism which is not necessary here. In a particular case of the integrable $V_{4}^{3}$ distribution, both the tetrad and monad methods determine the spinors in terms of the intrinsic geometry of foliation.

We ascertain the appearance of additional differences between the evolutions of the Weyl, Dirac and Maxwell fields, since the interaction of these fields with the inertial field is described by the term which includes not only the angular velocity vector of the frame of reference, but also its angular velocity.

\section{References}

1. Sommers P. Space spinors. // J. Math. Phys., 1980, vol. 21, No 10, p. 2567-2571.

2. Sen A. On the existence of neutrino "zero-modes" in vacuum spacetimes. // J. Math. Phys., 1981, vol. 22, No 8, p. 1781-1786.

3. Ashtekar A., Horowitz G. T. Phase space of general relativity revisited: A canonical choice of time and simplification of the Hamiltonian. // J. Math. Phys., 1984, vol. 25, No 5, p. 1473-1480.

4. Reula O. Existence theorem for solutions of Witten's equation and nonnegativity of total mass. // J. Math. Phys., 1981, vol. 22, No 8, p. 810-814.

5. Frauendiener J. Triads and the Witten equations. // Class. Quantum Grav., 1991, vol. 8 , No 10, p. 1881-1887. 
6. Pelykh V. The spinors, associated with $V_{4}^{3}$ distributions and generalization of SenWitten equation. // Mathematical methods and phisicomechanical fields, 1996, vol. 39, No 2, p. 133-139 (in Ukrainian).

7. Penrose R., Rindler W. Spinors and space-time. Vol. 1. Cambridge, Cambridge University Press, 1984.

8. For the expressions written in coordinates, Greek indices are global and Latin ones are local. Our Lorentz metric will have the signature $(+2)$

\section{Квадровані поля Вейля і Дірака із зв'язністю Соммерса-Сена, асоційованою із розподілами $V_{4}^{3}$}

\section{В.Пелих}

Інститут прикладних проблем механіки і математики

ім. Я.С.Підстригача НАН України, 290601 м. Львів, вул. Наукова, $3^{\sigma}$

Отримано 10 лютого 1998 р.

На основі узагальнення спінорної зв'язності Соммерса-Сена для полів, асоційованих із розподілом $V_{4}^{3}$, отримано рівняння для нульових полів Вейля і Дірака у комплексифікованому розподілі $V_{4}^{3}$. Отримані рівняння дозволяють описати взаємодію нульових полів із полями інерції.

Ключові слова: спінор, многовид, розподіл, нульове поле

PACS: $04.20 .-q, 02.40 . K y$ 